\title{
La Paradiplomacia Universitaria: La internacionalización de la educación superior en América
} University Paradiplomacy: The internationalization of Higher Education in latin America

David Horacio, García Walman ${ }^{1}$ Universidad Autónoma de Nuevo León

https://orcid.org/0000-0002-0623-4874

Paulina, Jiménez Quintana² Universidad Autónoma de Nuevo León https://orcid.org/0000-0003-3460-5668

María Gabriela, Zapata Moran ${ }^{3}$ Universidad Autónoma de Nuevo León

DOI: http://dx.doi.org/10.29105/pgc4.8-3 (D) https://orcid.org/0000-0002-4386-084X

\section{RESUMEN}

El presente artículo es producto de una revisión bibliográfica, cuyo objetivo consiste en analizar el impacto que las universidades tienen como agentes de Paradiplomacia en la esfera internacional a través de sus procesos y estrategias de internacionalización para lo cual se tomó el caso de algunas Universidades de tres países latinoamericanos, Chile, Argentina y Brasil. Se aplicó el método de análisis, con un enfoque cualitativo, diseño no experimental, bajo un nivel documental-bibliográfico transversal. Tras la revisión documental se realiza un breve análisis en el cual se encontraron diferentes estrategias de internacionalización, así como los procesos históricos que llevaron a las Universidades a expandirse. Se concluye que la internacionalización de la educación superior en América Latina sigue enfrentando retos, sin embargo, las estrategias implementadas por estos países han lanzado a sus Universidades al mercado internacional.

Palabras clave: Internacionalización de la educación, Latinoamérica, movilidad estudiantil, paradiplomacia.

\section{ABSTRACT}

This article is the product of a literature review, which objective is to analyze the impact that universities have as agents of Paradiplomacy in the international sphere through their internationalization processes and strategies for which the case of some Universities of three Latin American countries, Chile, Argentina and Brazil were taken. The method of analysis was applied, with a qualitative approach, non-experimental design, under a transversal documentary-bibliographic level. After the documentary review, a brief analysis is carried out in which different internationalization strategies were found, as well as the historical processes that caused the Universities to expand. It is concluded that the internationalization of higher education in Latin America continues to face challenges, however, the strategies implemented by these countries have launched their universities to the international market.

Key words: Internationalization of education, Latin America, student mobility, paradiplomacy.

Cómo referenciar este artículo:

García Walman, D., Jiménez Quintana, P., \& Zapata Moran, M. (2018). La Paradiplomacia Universitaria: La internacionalización de la educación superior en América. Revista Política, Globalidad y Ciudadanía, 4(8), 37-48. Recuperado de http://revpoliticas.uanl.mx/index.php/RPGyC/article/view/101

Recibido: 11 e Enero 2018 - Aceptado: 02 de Marzo 2018

\section{(oc) BY-NC-ND}

1 Doctor en Filosofía con orientación en Relaciones Internacionales, Negocios, y Diplomacia. . Email: david. garciaw@uanl.mx Orcid ID: https://orcid.org/0000-0002-0623-4874

2 Maestra en Ciencias del Emprendimiento y la innovación para un mundo sostenible. Email: paulina.jimenezq@uanl.edu.mx. Orcid ID: https://orcid.org/0000-0003-3460-5668

3 Doctorando en Filosofía con orientación en Relaciones Internacionales, Negocios, y Diplo.macia. Email: gabrielazapmor15@gmail.com. Orcid ID: https://orcid.org/0000-0002-4386-084X

Revista Política, Globalidad y Ciudadanía, Vol. 4 No. 8, Julio - Diciembre 2018, Universidad Autónoma de Nuevo León, Monterrey, México, ISSN 2395-8448. pp 37-48. http://revpoliticas.uanl.mx/index.php/RPGyC/article/view/101 


\section{INTRODUCCIÓN}

La educación superior se encuentra inmersa en un ambiente altamente competitivo a nivel internacional; sin embargo, a través del tiempo la colaboración (o cooperación) ha dejado en claro las ventajas que conlleva para las mismas. El éxito de estos esfuerzos de colaboración y la cooperación depende de una serie de factores, y una preocupación central de estos acuerdos y su potencial para persistir en la medida en que los problemas de autonomía institucional pueden abordar el éxito.

Los programas de intercambio han forzado el contacto entre diferentes sistemas educativos en diferentes países y han constituido el primer paso en los cambios en la educación superior que nacieron con la Declaración de Bolonia. Esta realidad se debe al hecho de que podemos apreciar cómo las habilidades de comunicación interculturales y multilingües ofrecen a los profesionales mejores oportunidades de trabajo y ventajas competitivas para sus negocios (Rondeau \& Dejanon , 2016).

En la presente investigación se plantea el papel de las universidades latinoamericanas en el proceso de internacionalización y como estos esfuerzos y estrategias han creado un ambiente de diplomacia alternativa, que los lleva a exponer y potencializar sus regiones o países.

La paradiplomacia cobra un sentido importante en el contexto de la internacionalización de las universidades, pues es a través de estas relaciones internacionales paralelas que el profesional y el investigador fortalecen sus capacidades para el futuro.

\section{2.- FUNDAMENTO TEÓRICO}

\section{Paradiplomacia Universitaria: Convenios y acreditaciones}

En 1961, el historiador diplomático Rohan Butler definió "paradiplomacia" como el ejercicio de diplomacia personal y paralela que complementa o compite con la política exterior regular del gobierno (Butler, 1961). Aunque excluyendo cualquier forma de agencia gubernamental, esta definición conlleva las connotaciones que hacen de la "paradiplomacia" un concepto polémico hoy en día. También está presente la consideración de la paradiplomacia como una forma no gubernamental de mediación alternativa, ahora fomentada significativamente por las nuevas tecnologías de los medios.

Por su parte, Cornago (2000) define a la paradiplomacia como la participación de gobiernos no centrales en las relaciones internacionales a través del establecimiento de contactos ad hoc con entidades privadas o públicas del extranjero, con el fin de promover asuntos socioeconómicos y culturales, así como cualquier otra dimensión externa de sus competencias constitucionales. Por otro lado, Duchacek (2009) hace una clasificación entre la paradiplomacia directa e indirecta. "La paradiplomacia directa se da cuando la región o subgrupo tiene una actuación directa en la escena internacional y tiene las verdaderas relaciones al exterior. En cambio la paradiplomacia indirecta, se usa cuando desde el interior se hace presión para moldear las políticas que usara el gobierno central al exterior" ( p. 20).

Es importante para esta investigación definir la Paradiplomacia, pues es una via en que las Universidades pueden crear convenios que después se pueden extrapolar entre los Estados como regiones, por lo tanto, la internacionalización de la educación puede en este sentido ser parte del proceso de la Paradiplomacia indirecta.

La educación superior es la base del desarrollo del recurso humano del país, por lo que su papel en la internacionalización representa un pilar crucial en el desarrollo de la competitividad del Estado. También se habla de las relaciones de los gobiernos no centrales con centros culturales, de otros Estados incluyendo

Revista Política, Globalidad y Ciudadanía, Vol. 4 No. 8, Julio - Diciembre 2018, Universidad Autónoma de Nuevo León, Monterrey, México, ISSN 2395-8448. pp 37-48. http://revpoliticas.uanl.mx/index.php/RPGyC/article/view/101 
relaciones con los gobiernos de Estados extranjeros y se distingue de la diplomacia clásica que es específica sectorialmente, más que en representar a toda una unidad territorial (Iglesias \& Zubelzú, 2008).

De igual manera Iglesias y Zabelzú (2008) hablan de la peculiaridad de cada institución o región y la importancia de hacer estudios diferentes en ellas, la internacionalización no ha sido un proceso equitativo y por lo tanto es posible identificar notables diferencias en los perfiles de gestión, la capacitación en recursos humanos y los diseños institucionales que contribuyan a una efectiva gestión internacional.

Aunque existen autores como Ivo Duchacek (1984) y Soldatos Panayotis (1988) que remarcan que las Universidades no pueden ser parte del fenómeno de la Paradiplomacia, existen estudios como el de Martins Senhoras (2009) que no solo habla de ciertos actores fuera del Estado central, sino que también incluye también a todos los actores que tienen una actividad internacional, siendo o no estatales; así, las empresas o las universidades pueden hacer paradiplomacia pues emplean ciertos elementos que son fundamentales del concepto.

Pues, aunque existen estrategias por parte de las universidades, todavía hay mucho que hacer en cuanto al tema de internacionalización de la educación superior y del papel de las universidades en la cooperación internacional en sus regiones. Ruiz (2014) lo establece de la siguiente manera: "No se trata solo de enviar alumnos y docentes sino también de recibir, de generar convenios, hacer investigaciones no solo a un nivel local o de país si no en cooperación con otro país que puede ser de beneficio para ambos. El principal reto es la conciencia sobre la importancia de la internacionalización de la educación, muchas instituciones en América Latina y el Caribe no reconocen el valor en traer estudiantes extranjeros a sus instituciones, no comprenden aún que cuando esto se da, se genera ya una habilidad en múltiples grados, una multiculturalidad y un cambio paulatino en la cultura universitaria." (p.54).

La fuga de cerebros es otro de los problemas a los que se enfrenta la educación superior en América Latina, en la tabla 1 se muestra la relación entre los estudiantes nacionales adscritos a la Universidad Autónoma de Nuevo León y realizan una estancia o intercambio en el extranjero y los estudiantes extranjeros que ingresan a la universidad de igual manera.

La OCED definió en 2005 a la educación superior como un nivel o una etapa de estudios posterior a la educación secundaria. Dichos estudios se adelantan en instituciones de educación terciaria, como universidades públicas y privadas, institutos de educación superior y politécnicos, así como en otros tipos de escenarios como escuelas de secundaria, sitios de trabajo, o cursos libres a través de la tecnología informática y gran variedad de entidades públicas y privadas.

En concreto, para los fines de esta investigación las acciones internacionales en materia cultural y educativa que lleva a cabo las universidades donde se incluyen dentro de la llamada diplomacia pública específicamente en su componente cultural (diplomacia cultural), en virtud de que éstas se dirigen a públicos en el exterior entre los que se consideran universidades, cuerpos académicos, institutos de investigación, comunidades de diásporas, gobiernos, empresas, comunidades culturales, entre otros.

Para el caso de las motivaciones que originan el quehacer internacional de las universidades, consideremos que para Duchaceck (1990), éstas, sus académicos, los think tanks, los medios de comunicación, así como los líderes políticos, constituyen "grupos de presión" al interior del Estado que rompen con el supuesto de su unidad racional. En este sentido, al encontrarse al interior del Estado agentes con las condiciones suficientes para ser considerados actores internacionales, se pone en evidencia el ejercicio cotidiano de la puesta en marcha de una dimensión internacional multinivel.

Las universidades, como agentes sociales, han visto afectado su desarrollo, al igual que otros actores, 
debido a los procesos de la globalización y el avance de las tecnologías de la comunicación y la información, que poseen una incidencia negativa en la planificación y la política educativa (Law \& Pelgrum, 2004).

Tanto es así que muchas de estas instituciones se sujetan a procesos de certificación y acreditación internacional de sus planes educativos, internacionalizan sus currículos y ponen en práctica políticas de educación intercultural, estudios en el extranjero, así como el establecimiento de convenios con pares internacionales (Middlehurst, 2008).

Se parte de la idea de que las acreditaciones internacionales son importantes para fomentar la Paradiplomacia a través de la interacción de las instituciones evaluadoras y las Universidades, además de que es importante mencionar que las acreditaciones influyen en el ranking con el que está valorado las universidades y que a su vez es un factor importante en la decisión de los alumnos extranjeros.

\section{México}

La necesidad de algún proceso de acreditación surgió primero con problemas asociados con la definición institucional, vastas diferencias en la calidad institucional sin una base ampliamente aceptada para determinar la calidad y dificultades encontradas por estudiantes e instituciones en la transferencia de créditos (Bogue, 1992, p 36). Por su parte Davenport (2001) asevera que, en los últimos años, el cambio en el énfasis de la acreditación ha sido dramático. Antes de 1980, los estándares de acreditación se centraban principalmente en los procesos, procedimientos e insumos, que se cree forman estudiantes bien educados y preparados.

Los factores determinantes que han influido en la internacionalización de la educación en México y por ende en los gobiernos estatales provienen de recomendaciones internacionales realizadas principalmente por la UNESCO y sus manifiestos sobre educación superior (Declaración sobre la Educación Superior en América Latina y el Caribe, de La Habana en 1996 y la Declaración Mundial sobre la Educación Superior en el Siglo XXI de París en 1998) y por la Organización para la Cooperación y el Desarrollo Económicos (OCDE) con el programa de Gestión de las Instituciones de Educación Superior (IMHE): a través de ellos, los Estados, entre ellos México, se han comprometido a impulsar reformas a la educación superior encaminadas a mejorar su competitividad y la de sus instituciones educativas, así como establecer políticas para fortalecer la dimensión internacional de la educación superior (Angulo, 2015).

Hasta hace poco la Universidad Autónoma de Nuevo León, no había reportado esfuerzos importantes en cuanto a la acreditación de sus programas, hoy en día cada vez más programas son acreditados internacionalmente por distintos organismos para mejorar su calidad y ser más competentes en el catálogo internacional.

Los esfuerzos de la UANL, son recientes, en 2010, la Universidad Autónoma de Nuevo León no reportó Programas Educativos (PE) acreditados internacionalmente (UANL, 2010), es hasta 2011 cuando aparece el primer dato siendo seis los PE que contaban con dicha acreditación, esta cifra se ha quintuplicado en proporción ya que para 2015 son $32 \mathrm{PE}$ los que han alcanzado una acreditación internacional (UANL, 2015). Por lo que se refiere a los convenios de colaboración e intercambio académico con instituciones extranjeras firmados por año se observa que la cifra se duplicó para 2015. Este dato suena relevante por los beneficios que dicha gestión ha de reportar. Resultaría de interés conocer los términos en los que se elaboran y que acciones contemplan, así como analizar el impacto de los mismos.

A pesar de los logros y actividades mencionadas, se deben redoblar esfuerzos no solo en el área de la acreditación sino también en el área de la cooperación y las relaciones que se crean. A pesar de que se han llevado a cabo cambios desde el rediseño curricular y la incorporación de la movilidad estudiantil al interior de las instituciones, hasta las tareas de investigación y gestión en las universidades, aún es necesario 
mejorar los mecanismos de coordinación y de reconocimiento mutuo entre las instituciones para establecer un marco local regional e internacional para cooperación y colaboración (Buendía, 2012). Esto resulta importante para establecer mecanismos y estructuras que permitan la cooperación internacional y fomenten las relaciones internacionales de maestros, investigadores y estudiantes.

La cantidad y diversidad de estructuras es extensa: desde relaciones simples entre colegas de diferentes instituciones, hasta redes, asociaciones, alianzas, asociaciones, consejos, coaliciones, pactos, federaciones, colaboraciones y consorcios. La "cooperación interinstitucional" aparece en la literatura como un término general, capaz de incluir cualquier cosa, desde el simple intercambio de información hasta las estructuras mucho más formales de colaboración y consorcios (Bjork, 1984).

En este sentido, Berliner (1997) ofreció una definición de cuatro formas de asociación: trabajo en red, coordinación, cooperación y colaboración en ella caracterizó el trabajo en red como un intercambio muy informal de información, con una participación mínima de los demás. La coordinación es un poco más formal porque involucra a más personas, con un intercambio de ideas y algo de acceso a servicios y productos, pero las organizaciones siguen trabajando de manera independiente. El siguiente nivel, que se refiere a la cooperación, es una relación formal con organizaciones que trabajan juntas pero que aún conservan su autonomía.

Aunque los esfuerzos de la universidad autónoma de Nuevo León han sido de importancia, es necesario seguir fomentando la movilidad estudiantil y los convenios de cooperación, pues no se presenta un flujo constante de los mismos, como se puede apreciar en la siguiente tabla:

Tabla 1. Internacionalización de la UANL

\begin{tabular}{cccc}
\hline Año & Número de convenios & $\begin{array}{c}\text { Movilidad de estudiantes } \\
\text { nacionales }\end{array}$ & $\begin{array}{c}\text { Estudiantes extranjeros } \\
\text { recibidos }\end{array}$ \\
\hline $\mathbf{2 0 1 8}$ & 34 & 853 & 700 \\
$\mathbf{2 0 1 7}$ & 33 & 844 & 318 \\
$\mathbf{2 0 1 6}$ & 29 & 1109 & 631 \\
$\mathbf{2 0 1 5}$ & 50 & 1160 & 522 \\
$\mathbf{2 0 1 4}$ & 45 & 973 & 321 \\
$\mathbf{2 0 1 3}$ & 43 & 972 & 414 \\
$\mathbf{2 0 1 2}$ & 36 & 570 & 84 \\
$\mathbf{2 0 1 1}$ & 25 & 632 & 290 \\
\hline
\end{tabular}

Fuente: Elaboración propia, 2018.

Existen algunas universidades que han podido ejercitar la Paradiplomacia e impulsar su ciudad o región el caso más importante en el país es la Universidad Autónoma de México, de tal manera que ya cuenta con oficinas o sedes abiertas en otros países, hasta el año 2018 la Universidad Autónoma de México, contaba con 14 sedes en el exterior, de acuerdo al El coordinador de Relaciones y Asuntos Internacionales (CRAI) de la UNAM, Francisco Trigo En Estados Unidos se tienen dos escuelas de extensión en San Antonio y Chicago y tres centros de Estudios Mexicanos en Seattle, Los Ángeles y Tucson, por el lado europeo la sede abierta en Alemania se ubica en el edificio internacional de la Universidad Libre de Berlín, y también se tiene presencia en España, Francia e Inglaterra (2018). A estas se refiere el coordinador como "nuestras embajadas para apoyar a alumnos y profesores en esas naciones, para promover y facilitar el intercambio con otras universidades" (Trigo, 2018).

Por lo tanto, en un alto grado la UNAM promueve y ejecuta las funciones centrales de la paradiploma- 
cia, en la siguiente tabla se muestra la relación entre el número de estudiantes y el número de convenios internacionales firmados por año, como es posible observar en la siguiente tabla.

Tabla 2. Convenios y Movilidad UNAM

$\begin{array}{lccc}\text { Año } & \text { Número de convenios } & \begin{array}{c}\text { Movilidad de } \\ \text { estudiantes } \\ \text { nacionales }\end{array} & \begin{array}{c}\text { Estudiantes } \\ \text { extranjeros }\end{array} \\ \mathbf{2 0 1 8} & 14 & 1484 & 978 \\ \mathbf{2 0 1 7} & 123 & 382 & 970 \\ \mathbf{2 0 1 6} & 126 & 2,249 & 1,362 \\ \mathbf{2 0 1 5} & 198 & 3477 & 4318\end{array}$

Fuente: Elaboración propia, 2018.

Consecuentemente, la importancia de la paradiplomacia y la cooperación entre actores a nivel internacional no se ha incorporado de manera definitiva en las agendas gubernamentales ni tampoco en las políticas educativas de los gobiernos estatales de México, debido a la carencia de diversos elementos tales como recursos económicos, interés gubernamental, visión internacional y estrategia de vinculación internacional en materia de educación superior. Pese a la insuficiencia de estos elementos, en general, las entidades federativas cuentan con un marco jurídico educativo local que les permite actuar internacionalmente y también han establecido acuerdos interinstitucionales en este sector (Angulo, 2015).

El número de estudiantes de movilidad y de convenios firmados podría considerarse poco representativo en el caso de algunas universidades en México, por lo que hay que redoblar los esfuerzos en las estrategias de internacionalización, reformular planes de desarrollo que estén íntimamente relacionados con las características del lugar, de la universidad y de los objetivos a alcanzar.

Chile: La internacionalización después de la dictadura

Las Universidades en Chile han demostrado una participación mayor en la arena internacional en los últimos años, a pesar de que no hace mucho vivían oprimidas por una dictadura militar, lo cual las colocaba con un sesgo negativo en los medidores internacionales a diferencia del resto de Latinoamérica en cuanto a la exposición global y a la búsqueda de la excelencia incluso a nivel nacional.

La política internacional de la transición constituyó un determinante significativo del estilo transnacional en las relaciones exteriores. En adelante, los procesos políticos regionales ejercerían gran influencia en la definición de las prioridades de la política exterior, lo que afectaría el uso de determinados instrumentos políticos y diplomáticos, particularmente en el ámbito del multilateralismo (Wilhelmy, 2003).

De acuerdo a Wilhelmy (2003) en Chile, varias instituciones académicas y centros de estudio jugaron un rol relevante en la constitución de grupos de trabajo que aportarían antecedentes analíticos y prácticos para la confección de la política exterior de los gobiernos de la transición. Destacados actores de dicha política, como José Miguel Insulza, Heraldo Muñoz, Juan Somavía, Juan Gabriel Valdés, Carlos Portales y Alberto van Klaveren hicieron aportes esenciales desde la fase programática, basándose en sus relevantes conocimientos especializados. Posteriormente, varios de ellos asumirían altas responsabilidades oficiales.

Por otra parte, como un modo de reinsertar al país en el escenario internacional tras el aislamiento im- 
puesto por los 18 años de dictadura, los gobiernos democráticos, desde 1990, promovieron el fomento de iniciativas tendientes a fomentar la internacionalización de la educación superior (Castillo, 2016).

En el análisis de los estudiantes que deciden estudiar en América Latina y el Caribe los estudiantes móviles provienen en su mayoría de dentro de la misma región: siete de cada diez alumnos extranjeros que estudian en alguna universidad latinoamericana son de origen latinoamericano (Geldres, 2013).

La academia es cada vez más internacional en sus alcances "los programas que impulsan la internacionalización en Latinoamérica son los intercontinentales como ALFA y COLOMBUS; convenios subregionales, como el Convenio Andrés Bello, Asociación de Universidades del Grupo de Montevideo (AUGM), para MERCOSUR; redes iberoamericanas (RIACES) y agencias regionales (IESALC). Cada vez son más las alternativas de postular a programas de diversos países, redes entre universidades regionales y mundiales que otorgan financiamientos becas a estudiantes latinoamericanos" (López, 2008).

Las políticas no tan sólo apuntan a incorporar la dimensión internacional en las actividades de índole académica sino también en las de administración y gestión, lo cual implica apoyar a las diversas instancias de las estructuras universitarias y los docentes para realizar contactos, para buscar pares internacionales y para obtener el financiamiento adecuado para llevar adelante sus proyectos y programas.

Respecto a la satisfacción de los estudiantes en relación a su experiencia de movilidad internacional, se identificó el idioma de origen afecta la relación de los estudiantes extranjeros con profesores y alumnos locales, impactando el grado de satisfacción con la experiencia total. En general, los estudiantes extranjeros tuvieron una buena percepción del programa y de su experiencia, fortaleciendo sus competencias académicas, personales e interculturales. Sin embargo, algunos manifestaron su disconformidad en relación con algunos profesores y estudiantes locales, como también con los paros estudiantiles y los servicios de hospedaje de bajo nivel en las dos ciudades donde vivieron. El estudio sugiere que las universidades en destinos emergentes deben capacitar a sus profesores y alumnos para integrar a los estudiantes extranjeros, así como agregar servicios de hospedaje para satisfacer las necesidades de éstos (López, 2008).

En este contexto, el proyecto "Consolidación de la Internacionalización de la Investigación y Postgrado de la Universidad de Chile" surge como una oportunidad de reforzar el enfoque internacional en la institución y constituirse en un polo relevante para el desarrollo de la investigación y la formación doctoral a nivel regional y global, ofreciendo alternativas formativas para jóvenes talentos provenientes de otros países, capacidades instaladas para llevar adelante proyectos de investigación cooperativa y para impulsar el desarrollo de redes internacionales de cooperación (Chile, 2019).

Argentina: Una política exterior local

La internacionalización de la educación superior es una de las tendencias clave del desarrollo. Existen varios enfoques sobre cómo lograr la competitividad y el rendimiento en la educación superior y la movilidad académica internacional; programas de intercambio de estudiantes, asociaciones son algunos de los aspectos que pueden desempeñar un papel importante en este proceso.

En Latinoamérica se han creado redes de cooperación que han ayudado a fortalecer las relaciones entre las Universidades y a promover las regiones de una manera más local. A finales de la década de los 90 , se crea el Programa de Desarrollo del Sector Educativo del MERCOSUR, el cual amplió las bases para el desarrollo del proceso de internacionalización de la educación superior, mediante la configuración de políticas regionales en materia educativa, que vienen a instalar la internacionalización de la universidad como asunto prioritario para los gobiernos, instituciones y actores universitarios (Ramírez, 2017). En un contexto internacional, donde está cambiando la arquitectura de la cooperación, resurgen programas de Cooperación

Revista Política, Globalidad y Ciudadanía, Vol. 4 No. 8, Julio - Diciembre 2018, Universidad Autónoma de Nuevo León, Monterrey, México, ISSN 2395-8448. pp 37-48. http://revpoliticas.uanl.mx/index.php/RPGyC/article/view/101 
Sur-Sur como instrumentos que invitan a elaborar proyectos de desarrollo endógeno, de características horizontales y fomento de la integración regional (Oregioni, 2015).

En Argentina, los gobiernos vienen impulsando desde el año 2003 líneas de acción y financiamiento en materia de políticas públicas de fomento de la internacionalización que han potenciado las actividades de cooperación de las internacionales de las universidades y ha impulsado su posicionamiento en la región (Astur \& Larrea, 2012).

Ramirez (2017) habla del caso en Argentina, en el cual se menciona que la internacionalización de la educación superior como tema de agenda de políticas educativas, viene a instalarse en Argentina hacia el año 2000, en un contexto nacional e internacional con tendencias que favorecen la integración y la cooperación entre los países. Frente a este escenario, indagó sobre cuáles han sido las implicancias de las acciones de internacionalización en las últimas décadas en las universidades argentinas y en qué medida estos procesos han colaborado o contrarrestado a la mayor dependencia académica o al despliegue de estrategias al interior de las instituciones de educación superior, tendientes a la internacionalización en casa.

A partir de la primera década del siglo XXI, cuando en el país se comienza a configurar un dispositivo político, desde el Ministerio de Educación a través de la Secretaría de Políticas Universitarias, con la implementación de programas que fomentan la internacionalización de las universidades argentinas como Universidad Nacional de La Pampa y la Universidad Nacional de las Artes, se viabilizó en mayor o menor grado la inclusión y la participación de todas las universidades nacionales; Esto, a su vez, generó un proceso dinámico y cambiante al interior de las instituciones, en el que algunas universidades se alinearon de una manera más proactiva, otras se adecuaron al nuevo contexto de forma más reactiva y otras en algunos casos excepcionales se vieron fraccionadas por encuentros y desencuentros de los propios actores, que asumieron posiciones antagónicas ante este proceso (Ramírez, 2017).

La internacionalización de la educación superior a nivel nacional e institucional en cualquier país debe entenderse como el proceso de integración de una dimensión internacional o intercultural internacional dentro de las funciones principales del sistema educativo. En el caso argentino, crearon un proceso en el que el gobierno estaba involucrado en el proceso de internacionalización de las Universidades a través de políticas de cooperación.

En este marco, se ha tratado de mostrar que aquellas universidades que ya tenían incorporada en su proyecto institucional la esfera internacional, a priori de las políticas nacionales impartidas, sus acciones y estrategias institucionales en estos últimos años asumieron una posición más proactiva, que giró en torno a gestionar las actividades ya existentes, a las nuevas estructuras, funciones y roles internos creados, orientándose más hacia la búsqueda de un sentido propio, alineados a las tendencias de la internacionalización en casa y privilegiando la cooperación solidaria (Ramírez, 2017).

\section{Brasil: El Estado nacional y la educación superior}

Los sistemas nacionales de educación superior de los países en desarrollo como es el caso de Brasil y de los países de América Latina y el Caribe viene pasando a lo largo de las últimas tres décadas por un intenso proceso de transformación.

Este proceso multidimensional tiene que ver con la expansión de la matrícula en la educación superior, de manera convergente con la experiencia de otras regiones del mundo, como las de Norteamérica, Europa, Asia y África (Acevedo, 2004).

El debate sobre la enseñanza superior en Brasil atravesó el siglo XX acompañando el agravamiento de 
las asimetrías que ese sistema perpetúa como por ejemplo la masificación de la matrícula, ocurrida desde los gobiernos militares. En Brasil, la internacionalización de la educación superior viene cargada de tensiones entre una postura global y una postura local. Aunque la incidencia de la producción en el país sea pequeña, lleva una visión positiva y posibilita prever el crecimiento de la importancia de la internacionalización en la educación. Este hecho es acentuado por la fuerza del Estado en las determinaciones de la Enseñanza Superior en el país y se refleja en los marcos regulatorios nacionales (Acevedo, 2004).

La dimensión nacional refleja discusiones sobre la presencia del estado evaluador y, más recientemente, en cuanto al nivel internacional en contextos de liberalización de la educación. En el caso brasileño, retrata la fuerte presencia del estado nacional en la educación superior (Afonso, 2015).

Por ejemplo en el gobierno Lula Da Silva, la política externa mantuvo una postura multilateralista, con características de una política influenciada por la fuerte liderazgo personal del presidente, aunque existir la acción diplomático activo y dinámica de Celso Amorim, en el gobierno de Lula, Brasil aumento el perfil de la relaciones con la países africanos, con el fin de no solamente promover las relaciones económicas, sino también el rescate de la llamada la deuda humana, social y cultural (Vigevani, 2007).

En el caso específico brasileño, en términos comparativos entre los periodos de gobierno de Lula da Silva y el gobierno de Dilma Rousseff, se notó una secuencia en el aumento de alumnos en la enseñanza superior, teniendo en cuenta el aumento de instituciones de enseñanza superior en Brasil y los programas lanzados durante el período de gobierno Lula da Silva para acceso a la enseñanza superior, como el PROUNI y el REUNI, que se mantuvieron durante el gobierno de Dilma. Al analizar el número de alumnos que estudian en el extranjero, fue posible observar que no hubo una clara la diferencia en el flujo de estudiantes de movilidad (Piva, 2018).

La internacionalización de sus Universidades refleja posturas paradigmáticas de la formación de recursos humanos de alto nivel para competir en un mercado internacional en el que el tránsito por países desarrollados exige calificación. Representa también en menor escala la apertura a los países latinoamericanos y africanos en un paradigma de solidaridad (Moog, 2018).

Brasil se enfrenta a otro problema para su región y es el idioma pues son pocos los países que hablan portugués, y por otro lado las instituciones de enseñanza superior de alto nivel enfrentan la competencia de nuevas unidades; lo cual ha forzado, junto con el incumplimiento a adoptar mecanismos de control, el financiamiento externo en lo que no ha dejado de ser atendido generosamente por el Estado.

El funcionamiento del mercado producirá una cierta selección y las menos equipadas para el objetivo están desapareciendo con la misma velocidad que anuncian sus productos. Es notable el fortalecimiento de la enseñanza privada para la que migran profesores jubilados o con baja remuneración en la enseñanza pública. Este es un punto de difícil equilibrio dado los rumbos de la economía y las asimetrías sociales y regionales del país (Piva, 2018).

\section{3.- MÉTODO}

Diseño

El enfoque investigativo de la presente investigación es cualitativo, de acuerdo con Hernández, Batista y Fernández (2014) “Utiliza la recolección y análisis de los datos para afinar las preguntas de investigación o revelar nuevas interrogantes en el proceso de interpretación” (p.7).

Alcanzando un diseño no experimental "Que se realiza sin la manipulación deliberada de variables y en los que sólo se observan los fenómenos en su ambiente natural para después analizarlos” (Hernández, Batista y Fernández, 2014, p. 149).

Revista Política, Globalidad y Ciudadanía, Vol. 4 No. 8, Julio - Diciembre 2018, Universidad Autónoma de Nuevo León, Monterrey, México, ISSN 2395-8448. pp 37-48. http://revpoliticas.uanl.mx/index.php/RPGyC/article/view/101 
El alcance establecido es el exploratorio "emplean cuando el objetivo consiste en examinar un tema poco estudiado o novedoso” (Hernández, Batista y Fernández, 2014, p. 91).

Instrumentos

Para la construcción del marco teórico-conceptual del tema "La paradiplomacia Universitaria: La Internacionalización de la educación superior en América Latina." se consultaron un total de treinta y nueve referencias bibliográficas utilizándose como instrumento las ideas, argumentos y proyectos que fueron interpretados desde una perspectiva analítica y crítica.

Procedimiento

Con relación a la comprensión del problema de la investigación se recopilan fuentes secundarias de documentos académicos. En el marco de referencia se definen los conceptos básicos relativos a la paradiplomacia universitaria. Una vez recopilada y analizada la información se construye el documento objeto de este trabajo. Por último, se realizan las recomendaciones y conclusiones conforme a los objetivos trazados (Bascón et al, 2016, p. 39).

\section{4.- CONCLUSIONES}

Los retos de las Universidades en Latinoamérica ante la globalización y la internacionalización no son fáciles, pues luchan contra gobiernos fallidos o repletos de corrupción, el idioma y la falta de consciencia ante un panorama internacional, como lo fue revisado en los casos anteriores. De esta manera, encontramos dos dificultades principales: la primera y más importante para los macro actores sociales es que las universidades del hemisferio norte logran seguir manteniendo en funcionamiento por el gran número de estudiantes extranjeros que los países envían para estudiar en los países desarrollados; y la segunda es la posibilidad de generar intercambio de conocimiento, mejorando la formación de los estudiantes de los países en desarrollo, que generalmente son países con poca inversión en educación y que pagan para enviar una parte de sus jóvenes para formarse. En ese segundo caso, algunos jóvenes brillantes pueden no regresar a su país de origen, siendo capturados por los países desarrollados para formar parte de empresas, investigaciones, por lo que se encuentra un problema grave de fuga de cerebros, y las universidades latinoamericanas no se presentan atractivas para el desarrollo profesional de los estudiantes, sin embargo, los esfuerzos expuestos en este artículo son palpables.

La nueva economía y la nueva sociedad basada en el conocimiento deben rediseñar el sistema de educación superior sólo para proporcionar las habilidades apropiadas y las habilidades para afrontar los nuevos desafíos; las universidades deben comprender la nueva posición en la que se encuentran como receptores de estudiantes internacionales y el impacto positivo que eso tiene en sus regiones y países.

Por tal motivo, es importante que se asuma el reto y que existan las políticas de internacionalización necesarias y adecuadas tanto a nivel institucional como local para promover la diplomacia paralela que podría ser el resultado de la cooperación entre Universidades. 


\section{REFERENCIAS}

Acevedo, E. (2004). Internacionalização da Educação Superior no Brasil. ASSOCIAÇÃO DE UNIVERSIDADES AMAZÔNICAS .

Alfonso, A. (2015). uestões, objetos e perspetivas em avaliação. Avaliaçã. Campinas .

Angulo, P. (2015). La internacionalización de los gobiernos locales a través de la educación superior en México: el caso de Jalisco. RIMAC .

Astur, A., \& Larrea, M. (2012). Políticas de internacionalización de la educación superior y cooperación. Obtenido de Minisitios del Ministerio de Educación: http://portales.educacion.gov.ar/spu/ cooperacioninternacional/documentos-de-interes/

Beder, H. (1984). Realizing the Principles for successful collaboration . Jossey-Bas .

Berliner, B. (1997). What it takes to work together: the prom ise of educational partnerships. Wested .

Bertram, D. (2008). Likert Scales . Topic Report , 1-10.

Braslavsky, C., \& Cosse, G. (2006). Las actuales reformas educativas en américa latina: cuatro actores, tres lógicas y ocho tensiones. Red Iberoamericana de Investigación sobre Cambio y Eficacia Escolar .

Buendía, A. (2012). Educación superior y aseguramiento de la calidad en México . CINDA .

Butler, R. (1961). Paradiplomacy, in Sarkissian. Studies in diplomacy .

Camicia, S., \& Zhu , J. (2011). Citizenship education under discourses of nationalism, globalization and cosmopolitalism. Frontiers of Education in China .

Castillo, P. (2016). Internacionalización de la investigación y el postgrado de la Universidad de Chile. Universidad de Chile .

Chile, U. d. (2019). Uchile . Recuperado de http://www.uchile.cl/internacionalizacion

Cornago, N. (2000). Diplomacia, paradiplomacia redefinición de la seguridad mundial . Madrid : Marcial Pons.

Davenport, T. (2001). Working knowledge: How organizations manage what they now. Harvard Business School Press .

Duchaceck, I. (1990). Perforated sovereignties: toward a typology of new actors. Oxford : Oxford University Press.

Duchaceck, L. (2009). The territorial dimension of politics: within, among and across nations. New york : Greenwood Press.

Geldres, V. (2013). Internacionalización de la educación superior en Chile. Redalyc .

Iglesias, E., \& Zubelzú, G. (2008). Las provincias argentinas en el escenario internacional. Desafíos y obstáculos de un sistema federal. Buenos Aires : Programa de las Naciones Unidas para el Desarrollo.

Justman, M., \& Thisse, J. (1997). Implications of the mobility of skilled labor for local public funding of higher education. econpapers .

Leininger, J., \& Javidan, M. (2010). Global leaders, global mindsets. China Staff .

López, F. (2008). Tendencias de la Educación Superior en el Mundo y América. Revista Avaliação .

Revista Política, Globalidad y Ciudadanía, Vol. 4 No. 8, Julio - Diciembre 2018, Universidad Autónoma de Nuevo León, Monterrey, México, ISSN 2395-8448. pp 37-48. http://revpoliticas.uanl.mx/index.php/RPGyC/article/view/101 
Middlehurst, R. (2008). Not Enough Science or Not Enough Learning? Exploring the Gaps between Leadership Theory and Practice . Higher Education Quarterly .

Moog, M. (2018). Internacionalização da educação superior. Creative Commons .

Obledo, G. A. (2016). La acción internacional en materia cultural y educativa de la universidad de Guadalajara y su relacion con la Paradiplomacia. . Madrid : Universidad Complutense de Madrid .

Oregioni, M. (2015). “Internacionalización de las Universidades Argentinas hacia la Región Latinoamericana a partir de Redes de Cooperación. UNICEN .

Piva, C. D. (2018). Análise da internacionalização da educação. International Journal of Higher education .

Ramírez, B. (2017). ¿Internacionalizar? ¿Por qué? ¿Para qué? La internacionalización en las. Debate Universitario .

Ruiz-Gutierrez, C. (2014). Internacionalización de la educación superior paradiplomacia universitaria- y la gestión de la comunicación en este proceso. Piura Peru: Universidad de Piura.

Rumbley, L. (2009). Trends in Global Higher Education:Tracking an Academic Revolution. UNESCO .

Sebastian, J. (2004). Cooperación e internacionalización de las universidades. biblo .

Shultz, L., \& Jorgenson, S. (2009). Global Citizenship Education in Post-Secondary Institutions: A Review of the Literature. University of Alberta .

Tardo, F. (2017). Experiencias y resultados preliminares sobre la nueva estrategia de idioma ingles. MEDISAN .

Teagarden, M. (2009). Developing globally competent leaders: The role of global mindset . Concepts and Connections .

Trigo, F. (21 de 06 de 2018). Ya suma la UNAM 14 sedes académicas en el extranjero. excelsior .

UNESCO. (1998). La educación superior en el siglo XXI. Conferencia Mundial sobre la Educación Superior .

Uribe, J. (2005). Internacionalización de la educación superior . Redalyc .

Vigevani, T. (2007). A política externa de Lula da Silva: a estratégia da. Contexto Internacional.

Wilhelmy, M. (2003). Los principales rasgos de la politica exterios chilena. Revista de ciencia política . 